\title{
GADD34 Promotes Tumor Growth by Inducing Myeloid-derived Suppressor Cells
}

\author{
LINTAO LIU, SACHIKO ITO, NAOMI NISHIO, YANG SUN, YURIKO TANAKA and KEN-ICHI ISOBE
}

Department of Immunology, Nagoya University Graduate School of Medicine, Nagoya, Japan

\begin{abstract}
Background: Tumor hypoxia induces the expression of growth arrest and DNA damage-inducible protein (GADD34). However, the role of GADD34 in tumor growth remains unclear. Materials and Methods: Gadd34 expression was knocked-down through lentivirus-mediated short hairpin RNA (shRNA) in tumor cells, which were subsequently injected subcutaneously into mice. Tumor volumes and myeloid-derived suppressor cells (MDSCs) were monitored. Isolated MDSCs were incubated with tumor supernatant to investigate the impact of GADD34 on cytokine secretion of MDSCs. Results: We observed that reduction of GADD34 expression significantly suppressed tumor, and resulted in decreased accumulation of MDSCs and T-cells, and inhibition of GADD34 reduced secretion of vascular epithelial growth factor $\alpha$ and transforming growth factor $\beta$ by MDSCs. Conclusion: These findings provide a promising strategy for targeting GADD34 activity in order to inhibit tumor growth.
\end{abstract}

Myeloid-derived suppressor cells (MDSCs) are heterogeneous populations of immature myeloid cells, which consist of two major subsets, monocytic $\mathrm{CD} 11 \mathrm{~b}^{+} \mathrm{Ly} 6 \mathrm{G}^{-} \mathrm{Ly} 6 \mathrm{C}^{\mathrm{hi}}$ and granulocytic $\quad \mathrm{CD} 11 \mathrm{~b}^{+} \mathrm{Ly} 6 \mathrm{G}^{+} \mathrm{Ly} 6 \mathrm{C}^{\text {low }} \quad$ (1-3). The immunosuppressive mechanism of MDSCs that suppress immune surveillance is considered to present by inhibiting Tcell-mediated antitumor activity (4-6).

As solid tumors grow, they inevitably experience a variety of stresses, including hypoxia, and reduced glucose and amino acid supply (7), all of which could contribute to the expression of growth arrest and DNA damage-inducible protein $(\mathrm{GADD} 34)(8,9)$. These findings prompted us to

Correspondence to: Lintao Liu, Department of Immunology, Nagoya University Graduate School of Medicine, 65 Turumai-cho, Showa-ku, Nagoya, Aichi, 466-8550, Japan. Tel: +81 527442135, Fax: +81 527442972, e-mail: Liulintao2013@Gmail.com

Key Words: GADD34, Gr1+CD11b+ MDSC, Lewis lung carcinoma, VEGFA, TGF $\beta$. hypothesize that GADD34 should be involved in tumor growth. In the present study, we explored the mechanism of action of GADD34 on tumor growth.

\section{Materials and Methods}

Cell culture. Lewis lung carcinoma (LLC) cells (ATCC, Rockville, MD, USA) were routinely grown in DMEM medium supplemented with $10 \%$ fetal bovine serum. GADD34 was knocked-down through lentivirus-mediated shRNA (Sigma Aldrich, St Louis, MO, USA) according to our previous report (10). The reduction of GADD34 was determined by stimulating LLC cells with MG132 (Calbiochem, San Diego, CA, USA). In order to mimic a hypoxic environment, $\mathrm{CoCl} 2$ was used to treat LLC cells.

Tumor model. Eight-week-old C57BL/6 and KSN nude female mice were purchased from SLC (Sizuoka, Japan). Animal work was carried out in the Animal Research Facility at the Nagoya University Graduate School of Medicine in accordance with institutional guidelines. LLC cells $\left(1 \times 10^{6}\right)$ were injected subcutaneously and tumor volumes were monitored for 18 days in C57BL/6 mice or 20 days in KSN mice, and determined using the formula $V=\pi / 6 \times$ length $\times$ width (2).

Flow cytometry. Cells from spleens, bone marrows, and peripheral blood were labeled with phycoerythrin (PE)-anti-granulocyte receptor-1(Gr1), fluorescein isothiocyanate (FITC)-anti-CD11b, PEanti-Ly6G, allophycocyanin (APC)-anti-Ly6C, APC-anti-CD4, FITC-anti-CD8 (BD Biosciences, Franklin Lakes, NJ, USA) and Fluro480-anti-CD11b and analyzed on a BD FACSCanto II Flow Cytometer (Becton Dickinson).

In vitro stimulation of MDSCs. LLC cells were cultured under normoxic or hypoxic (with $100 \mu \mathrm{M} \mathrm{CoCl}_{2}$ ) condition. The conditioned media were harvested centrifuged, and filtered. For in vitro stimulation, $\mathrm{Gr} 1{ }^{+} \mathrm{CD} 11 \mathrm{~b}^{+}$cells were isolated from the bone marrow of C57BL/6 mice using BD FACS Aria Cell Sorter (Becton Dickinson) after stained with PE-anti-Gr1 and Fluor480-antiCD11b.

Real-time polymerase chain reaction $(P C R)$. RNA isolation and Taqman reverse transcriptase reactions (Applied Biosystems, Foster City, CA, USA) were performed according to the manufacturer's instructions. Real-time PCR reactions (SYBR Green system) were conducted using murine primer sequences as follows: Gadd34: 
A

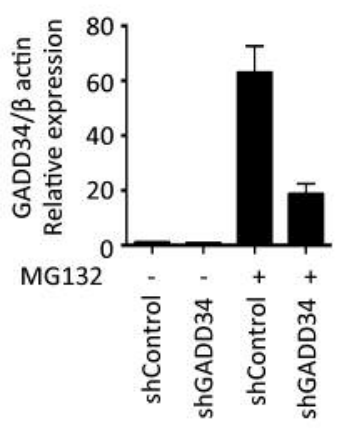

D

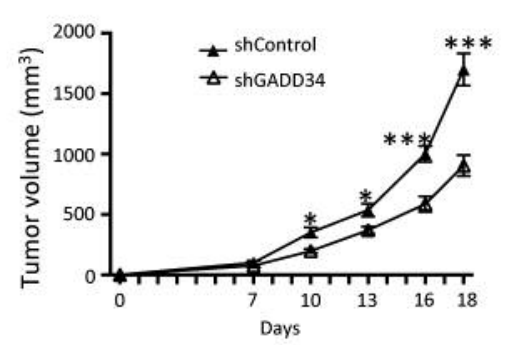

G

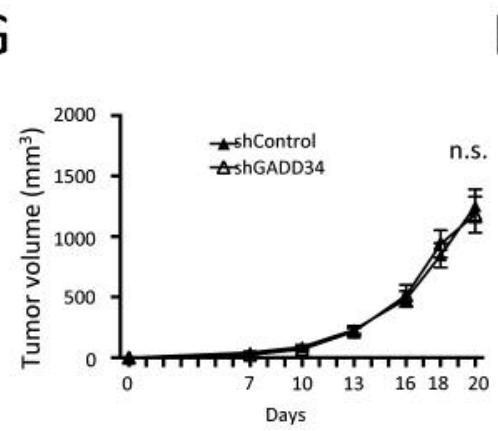

$E$

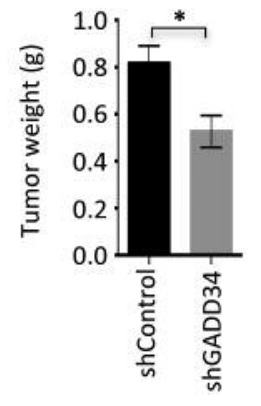

B

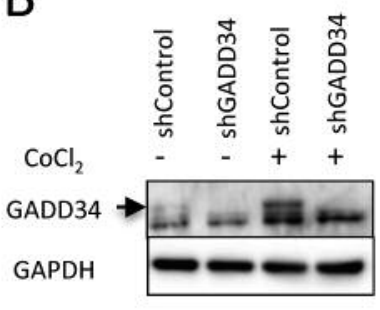

$\mathrm{H}$

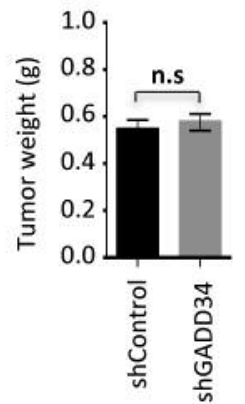

$\mathrm{F}$

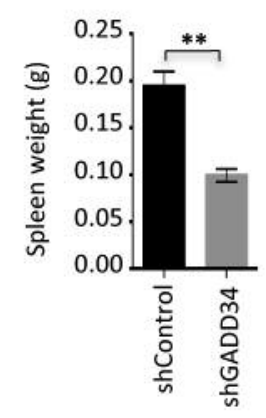

C
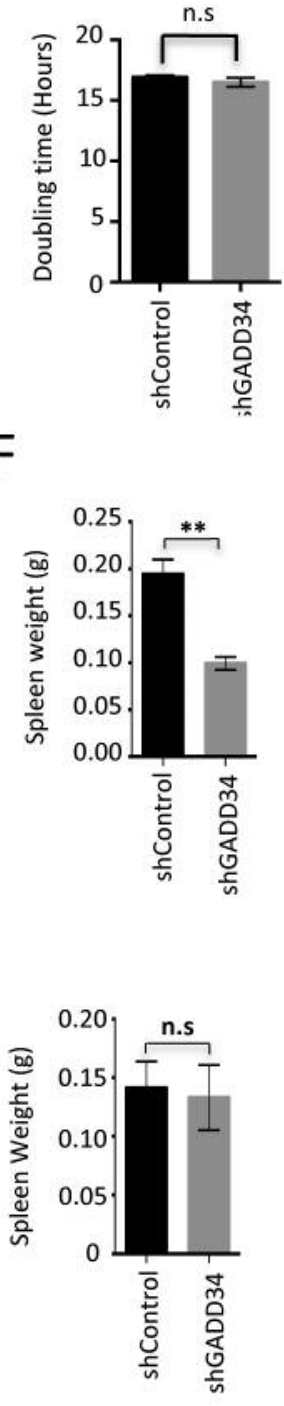

Figure 1. Short hairpin RNA for growth arrest and DNA damage-inducible protein (ShGADD34) tumor-bearing mice exhibited reduced tumor growth. Gadd34 expression was analyzed by real-time PCR in Lewis lung carcinoma cells following MG132 (10 $\mu M)$ stimulation for 24 h (A), and by western blot following 24-h $\mathrm{CoCl}_{2}(100 \mu \mathrm{M})$ treatment $(B)$. The data represent three independent experiments. C: Doubling time of shControl and shGADD34 cells under hypoxic condition. In C57BL/6 mice ( $D, E$, and $F)$, tumor growth was monitored for an 18-day period ( $n=5$ mice per group). In KSN nude mice $(G, H$, and I), tumor volume was monitored for a 20-day period ( $n=4$ mice per group). Then tumors and spleens were dissected from the mice. n.s. $p>0.05, * p<0.05, * * p<0.01$, and $* * * p<0.001$.

forward: 5' -aggacccegagattcctcta-3', reverse: 5'-aggtagggacccagcttctc3'; of transforming growth factor $\beta(T g f b)$ : forward: 5'ggactctccacctgcaagac-3', reverse: 5'-gactggcgagccttagtttg-3'; vascular epithelial growth factor $\alpha$ (Vegfa): forward: 5'-tgtacctccaccatgccaagt3', reverse: 5'-tggaagatgtccaccagg gt-3'; $\beta$-actin $(A c t b)$ : forward 5'agtgtgacgttgacatccgt-3', reverse: 5'-gcagctcagtaaca gtccgc-3'.

Statistical analysis. Cell doubling time was calculated using Doubling Time Online Calculator (http://www.doublingtime.com/compute.php). Statistical comparisons were performed by
Student's $t$-test to identify statistical significance. All data are shown as the mean \pm SEM. Significance was accepted at $p<0.05$.

\section{Results}

GADD34 promotes tumor growth in LLC tumor-bearing mice. To detect the efficacy of Gadd34 knockdown, cells were treated with MG132, a strong inducer of Gadd34. Quantitative PCR results showed $70 \%$ reduction of Gadd34 

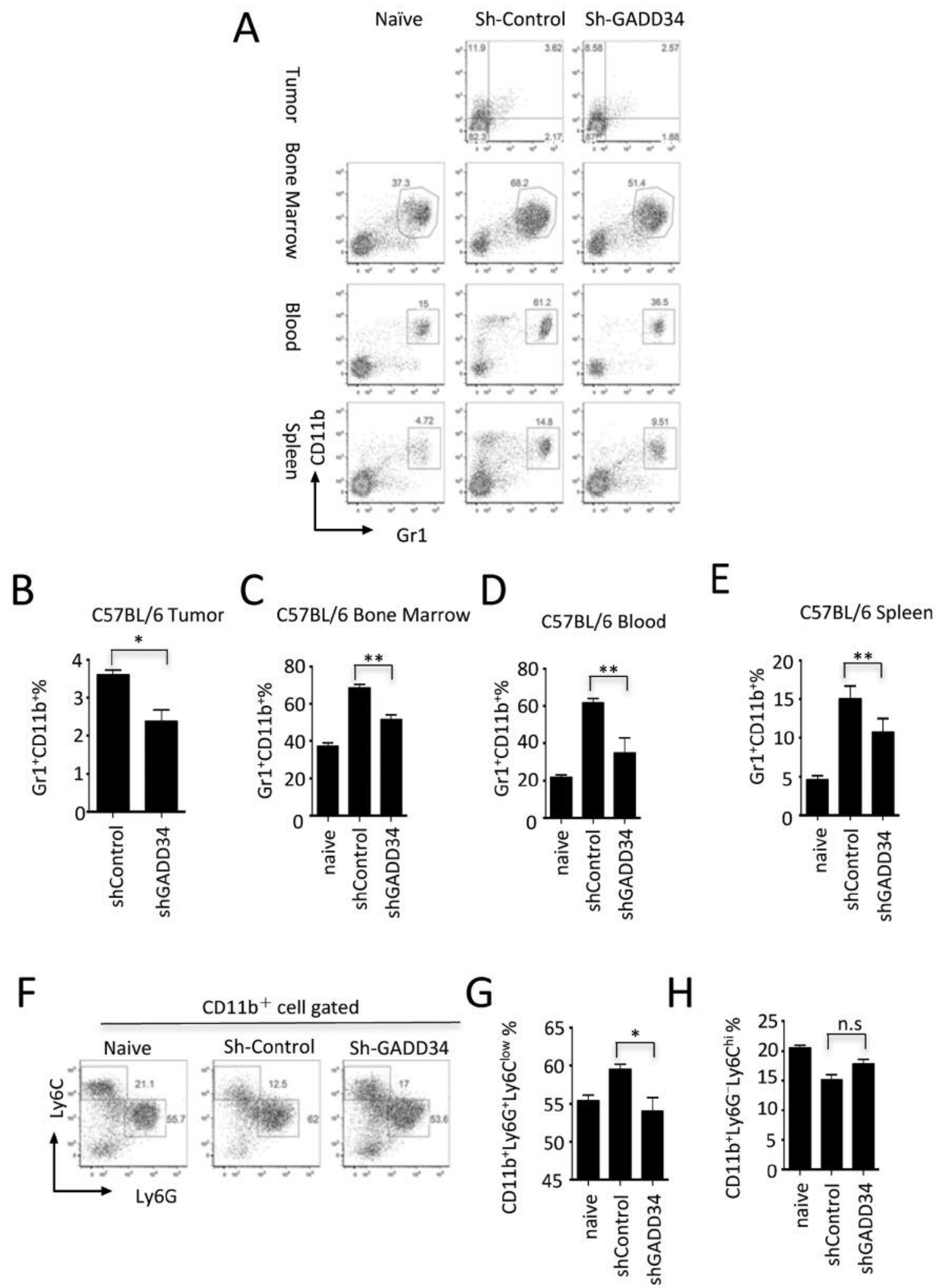

Figure 2. Accumulation of $\mathrm{Gr}^{+} \mathrm{CD} 11 b^{+}$myeloid cells. A: Typical example of flow cytometric analysis. The percentage of Gr1 ${ }^{+} \mathrm{CD} 11 b^{+}$cells in tumor $(B)$ bone marrow $(C)$, peripheral blood $(D)$ and spleen $(E)$ from $C 57 B L / 6$ mice 18 days after inoculation $(n=5$ mice per group) are shown. $F$ : Typical example of flow cytometric analysis. The percentage of $L y 6 G^{-} L y 6 C^{h i}(G)$ and $L y 6 G^{+} L y 6 C^{l o w}(H)$ cells in CD11b ${ }^{+}$cells of bone marrow ( $n=5$ mice per group). n.s. $p>0.05, * p<0.05$ and ${ }^{* *} p<0.01$. 


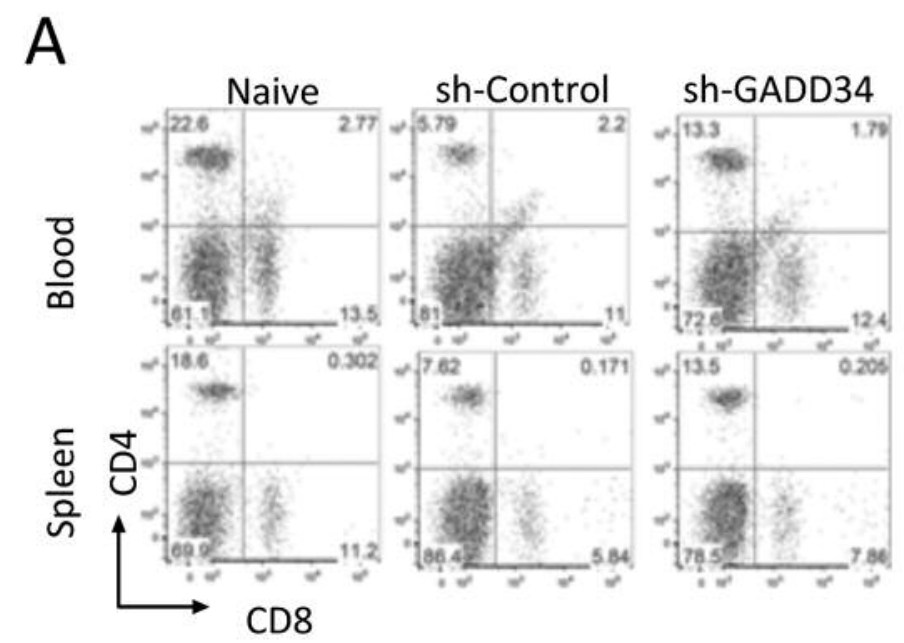

B

C C57BL/6 Spleen

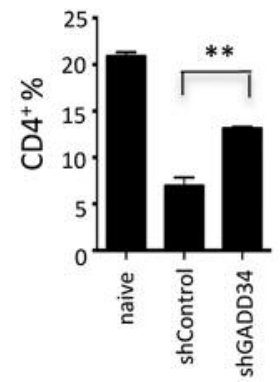

C57BL/6 Blood

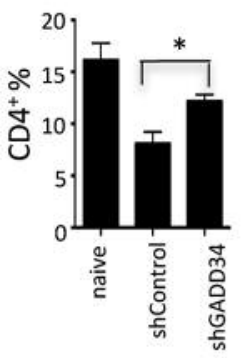

D

C57BL/6 Spleen

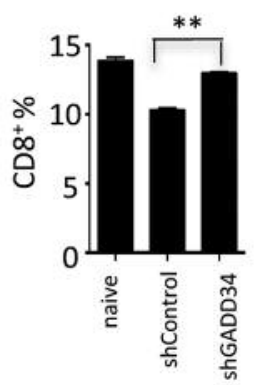

$\mathrm{E}$

Figure 3. Accumulation of T-cells. A: Typical flow cytometric plots of $\mathrm{CD}^{+}$, and $\mathrm{CD} 8^{+}$T-cells in peripheral blood and spleen. The percentage of $C D 4^{+}$cells in spleen $(B)$ and peripheral blood $(C)$, and the percentage of $C D 8^{+}$cells in spleen $(D)$ and peripheral blood $(E)$ from mice 18 days after injection of indicated Lewis lung carcinoma cells or naive cells into mice were assessed ( $n=5$ mice per group). n.s. $p>0.05, * p<0.05$ and $* * p<0.01$.

mRNA (Figure 1A). Similarly, hypoxia through $\mathrm{CoCl}_{2}$ treatment did not induce obvious expression of Gadd34 in shGADD34 cells (Figure 1B). Next, analysis of the doubling time of LLC cells indicated that there was no significant change after Gadd34 knockdown, and the mean value of both cell lines was around 17.3 h (Figure 1C).

In order to evaluate the role of Gadd34 in tumor growth, LLC cells were injected (s.c.) into the flanks of C57BL/6 and KSN nude mice. In C57BL/6 mice, we found that Gadd34 knockdown resulted in inhibition of tumor volume (Figure 1D), tumor weight (Figure 1E) and spleen weight (Figure 1F) 18 days after tumor inoculation. However, such inhibition was not observed in KSN nude mice (Figure 1GI). These data indicate that GADD34 is required for promoting tumor cell growth in vivo, and such promotion is T-cell dependent.
ShGADD34 tumor-bearing mice showed reduced accumulation of $\mathrm{GrI}^{+} \mathrm{CD} 11 b^{+} \mathrm{MDSCs}$ and T-cells. MDSC numbers are often correlated with tumor size. Therefore, we evaluated the presence of MDSCs in tumors, bone marrow, spleen and peripheral. Flow cytometric analysis showed that the extent of increase of $\mathrm{Gr}^{+} \mathrm{CD} 11 \mathrm{~b}^{+}$MDSCs in ShGADD34 tumor-bearing mice was lower than that in ShControl tumor-bearing mice (Figure 2A-E). Moreover, In shControl tumor-bearing mice, the proportion of granulocytic $\mathrm{CD} 11 \mathrm{~b}^{+} \mathrm{Ly} 6 \mathrm{G}^{+} \mathrm{Ly} 6 \mathrm{C}^{\text {low }}$ MDSCs was significantly increased, whereas that of CD11 ${ }^{+}$Ly $6 \mathrm{G}^{-}$Ly $6 \mathrm{C}^{\text {hi }}$ MDSCs showed no significant difference compared to shGADD34 tumorbearing mice (Figure 2F-H).

Next, we evaluated the numbers of T-cells because MDSCs promote tumor progression by suppressing T-cells. In tumorbearing mice, $\mathrm{CD}^{+}$and $\mathrm{CD} 8^{+} \mathrm{T}$-cell levels were significantly 

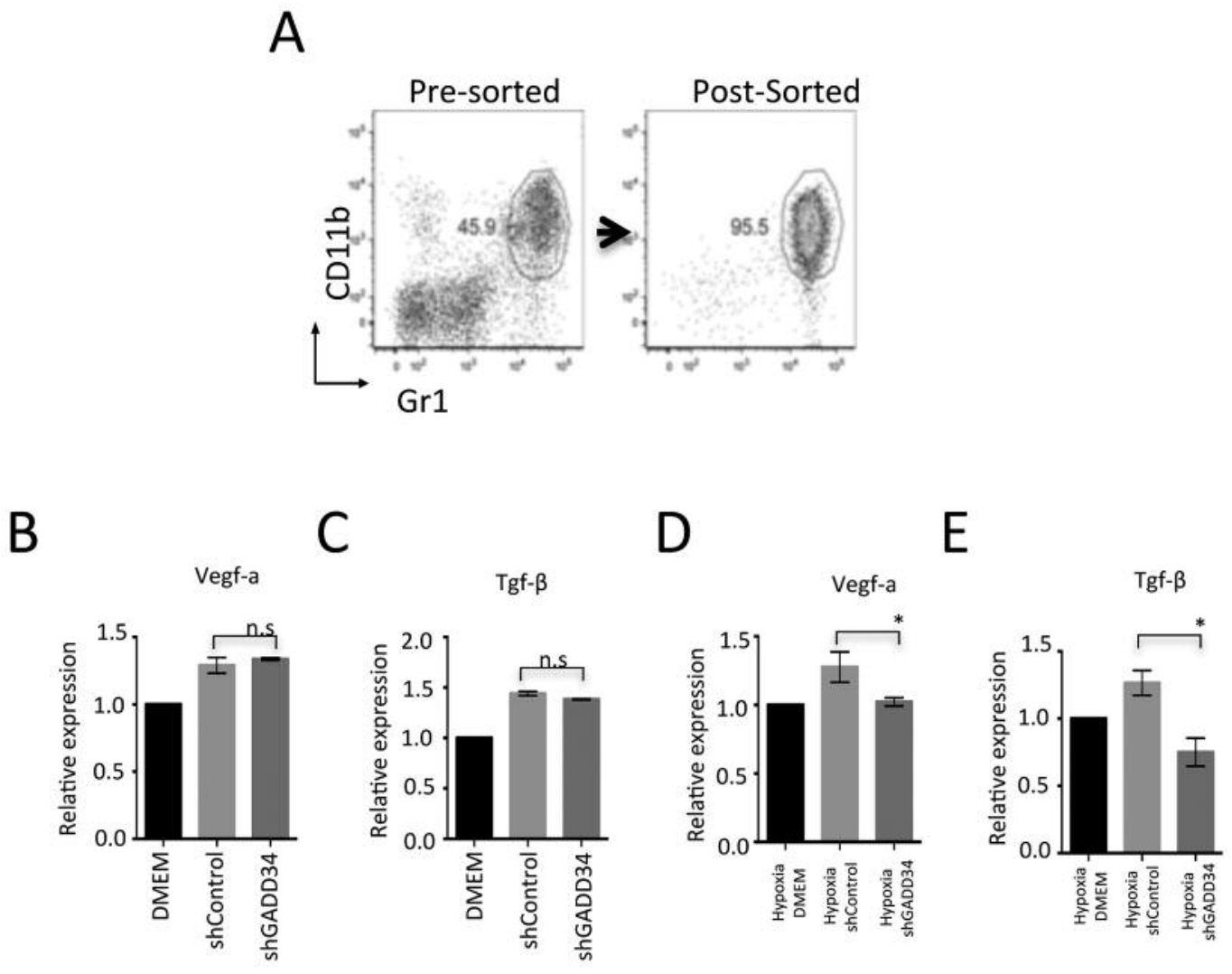

Figure 4. Tumor-conditioned medium induced vascular epithelial growth factor $\alpha$ (Vegfa) and transforming growth factor $\beta\left(\right.$ Tgfb) in Gr $1^{+} C D 11 b^{+}$ myeloid-derived suppressor cells (MDSCs). A: Typical example of sorting $\mathrm{Grl}^{+} \mathrm{CD} 11 \mathrm{~b}^{+}$cells from bone marrow of mice. Expression analysis by realtime polymerase chain reaction of Vegfa $(B)$ and Tgfb $(C)$ mRNA in $G r 1+C D 11 b^{+}$MDSCs stimulated with control medium (DMEM), control short hairpin RNA (shControl)- or sh DNA damage-inducible protein (GADD34)-conditioned medium under normoxic conditions (without $\left.C o C l_{2}\right)(B, C)$ or under conditions of hypoxia (with $100 \mu \mathrm{M}$ of $\left.\mathrm{CoCl}_{2}\right)(D, E)$ for 6 h. The results represent three independent experiments. $n . s . p>0.05$ and $* p<0.05$.

decreased compared to naive mice (Figure 3A). However, $\mathrm{CD} 4^{+}$and $\mathrm{CD} 8^{+} \mathrm{T}$ cells were reduced in spleens of shControltumor bearing mice (Figure 3B and D). In peripheral blood, shControl-tumor bearing mice also exhibited a significantly reduced level of $\mathrm{CD}^{+} \mathrm{T}$-cells (Figure $3 \mathrm{C}$ ), whereas $\mathrm{CD} 8^{+} \mathrm{T}$ cells showed no difference between these two types of LLC tumor-bearing mice (Figure 3E).

Gadd34 knockdown suppressed the secretion of VEGFA and $T G F \beta$. MDSCs suppress tumor growth mainly through producing VEGFA and TGF $\beta$ (18). Therefore, we hypothesized that levels of VEGFa and TGF- $\beta$ secreted by MDSCs should be affected by GADD34. The two types of cultured supernatant from shControl and shGADD34 cells were incubated with $\mathrm{Gr}^{+}{ }^{+} \mathrm{CD} 11 \mathrm{~b}^{+}$myeloid cells isolated from the bone marrow (Figure 4A). The results of real-time PCR indicated that under normoxic conditions, the expression of Vegfa and $T g f b$ in $\mathrm{Gr}^{+} \mathrm{CD} 11 \mathrm{~b}^{+}$MDSCs did not differ (Figure 4B and C). However, under hypoxic conditions, we observed higher expression of Vegfa and $T g f b$ (Figure 4D and $\mathrm{E}$ ) in $\mathrm{Gr}^{+} \mathrm{CD}^{+} 1 \mathrm{~b}^{+}$myeloid cells stimulated by LLC shControl tumor supernatant than those stimulated by LLC shGADD34 tumor supernatant.

\section{Discussion}

In this study, we identified accumulation of MDSCs as one novel mechanism by which GADD34 promoted tumor growth under hypoxic conditions. One major manner of tumor cell communication with MDSCs is by producing various factors [such as granulocyte macrophage colonystimulating factor, macrophage colony-stimulating factor, granulocyte colony-stimulating factor, VEGF $\alpha$, and TGF $\beta$ (11-13)] and small molecules [e.g. lactic acid (14)]. In this study, we showed expression of GADD34 up-regulated the secretion of VEGFA and TGF $\beta$ in MDSCs, which provides an explanation of why GADD34 promoted tumor growth. Knockdown of Gadd34 did not reduce the tumor size in 
KSN nude mice (Figure 2). This means that MDSCs affect tumor size in a T-cell-dependent manner, and T-cells, not MDSCs, are the major factor controlling to tumor growth.

Recently, a new strategy of targeting GADD34 activity to suppress tumor growth was developed. The effect of Guanabenz which acts as an inhibitor of the regulatory subunit of protein phosphatase 1, PPP1R15A/GADD34 (15), has been evaluated in a mouse model of human breast cancer and the results showed Guanabenz attenuated breast cancer tumor cell growth (16). Our study demonstrated that expression of GADD34 promoted growth of lung carcinoma and revealed the immunological mechanisms, which provides the theoretical basis for anticancer research by targeting GADD34 protein.

\section{Acknowledgements}

The Authors thank M. Tanaka for technical assistance with Cell Sorting experiments experiments as well as N. Oiwa for administrative assistance. This work was supported by 25293166 Grants-in-Aid for Scientific Research from Japan Society for the Promotion of Science.

\section{References}

1 Ostrand-Rosenberg S and Sinha P: Myeloid-derived suppressor cells: linking inflammation and cancer. J Immunol 182: 44994506, 2009.

2 Umansky V and Sevko A: Tumor microenvironment and myeloidderived suppressor cells. Cancer Microenviron 6: 169-177, 2013.

3 Gabrilovich DI and Nagaraj S: Myeloid-derived suppressor cells as regulators of the immune system. Nat Rev Immunol 9: 162174, 2009.

4 Bronte V, Apolloni E, Cabrelle A, Ronca R, Serafini P, Zamboni $\mathrm{P}$, Restifo NP and Zanovell P: Identification of a CD11b(+)/Gr$1(+) / C D 31(+)$ myeloid progenitor capable of activating or suppressing CD8(+) T cells. Blood 96: 3838-3846, 2000.

5 Gabrilovich DI, Velders MP, Sotomayor EM and Kast WM: Mechanism of immune dysfunction in cancer mediated by immature Gr-1+ myeloid cells. J Immunol 166: 5398-5406, 2001.

6 Mazzoni A, Bronte V, Visintin A, Spitzer JH, Apolloni E, Serafini P, Zanovello P and Segal DM: Myeloid suppressor lines inhibit T cell responses by an NO-dependent mechanism. J Immunol 168 : 689-695, 2002.
7 Gabrilovich DI, Ostrand-Rosenberg S and Bronte V: Coordinated regulation of myeloid cells by tumours. Nat Rev Immunol 12: 253-268, 2012.

8 Park HR, Tomida A, Sato S, Tsukumo Y, Yun J, Yamori T, Hayakawa Y, Tsuruo T and Shin-ya K: Effect on tumor cells of blocking survival response to glucose deprivation. J Natl Cancer Inst 96: 1300-1310, 2004.

9 Liu L, Nishio N, Ito S, Tanaka $\mathrm{Y}$ and Isobe K: Negative regulation of GADD34 on myofibroblasts during cutaneous wound healing. BioMed Res Int 2014: 137049, 2014.

10 Ito S, Tanaka Y, Oshino R, Aiba K, Thanasegaran S, Nishio N and Isobe K: GADD34 inhibits activation-induced apoptosis of macrophages through enhancement of autophagy. Sci Rep 5: 8327, 2015.

11 Barreda DR, Hanington PC and Belosevic M: Regulation of myeloid development and function by colony stimulating factors. Dev Comp Immunol 28: 509-554, 2004.

12 Casella I, Feccia T, Chelucci C, Samoggia P, Castelli G, Guerriero R, Parolini I, Petrucci E, Pelosi E, Morsilli O, Gabbianelli M, Testa U and Peschle C: Autocrine-paracrine VEGF loops potentiate the maturation of megakaryocytic precursors through Flt1 receptor. Blood 101: 1316-1323, 2003.

13 Soderberg SS, Karlsson G and Karlsson S: Complex and context dependent regulation of hematopoiesis by TGF-beta superfamily signaling. Ann Ny Acad Sci 1176: 55-69, 2009.

14 Colegio OR, Chu NQ, Szabo AL, Chu T, Rhebergen AM, Jairam V, Cyrus N, Brokowski CE, Eisenbarth SC, Phillips GM, Cline GW, Phillips AJ and Medzhitov R: Functional polarization of tumour-associated macrophages by tumour-derived lactic acid. Nature 513: 559-563, 2014.

15 Tsaytler P, Harding HP, Ron D and Bertolotti A: Selective inhibition of a regulatory subunit of protein phosphatase 1 restores proteostasis. Science 332: 91-94, 2011.

16 Hamamura K, Minami K, Tanjung N, Wan Q, Koizumi M, Matsuura N, Na S and Yokota H: Attenuation of malignant phenotypes of breast cancer cells through eIF2alpha-mediated down-regulation of Rac1 signaling. Int J Oncol 44: 1980-1988, 2014.
Received July 8, 2016

Revised August 2, 2016

Accepted August 4, 2016 